\title{
The effects of maze-arm length on performance in the radial-arm maze
}

\author{
MICHAEL F. BROWN \\ Villanova University, Villanova, Pennsylvania
}

\begin{abstract}
Rats trained in a 16-arm radial maze with arms half the standard length demonstrated extremely low, but above chance, choice accuracy (Experiment 1). Rats trained in a 12-arm maze with short arms demonstrated a substantially higher degree of adjacent-arm responding than did rats trained in the same maze with long maze arms and, when response stereotypy was disrupted by a forced-choice procedure, the short-arm group chose less accurately than the long-arm group (Experiment 2). In a 16-arm maze with 8 short arms and 8 long arms, there was a strong preference for short arms and no evidence for a difference in the ability to discriminate previ. ously visited arms from unvisited arms as a function of arm length, as measured by a twoalternative forced-choice procedure (Experiment 3). These results are interpreted as indicating that arm length affects a choice criterion, with a relatively lax criterion being applied to shorter arms.
\end{abstract}

In the now familiar radial-arm maze task (Olton $\&$ Samuelson, 1976), rats choose from among a number of maze arms connected to a central platform in a radially symmetric fashion, like the spokes of a wheel. Revisits to arms constitute errors. Rats rapidly learn to choose very accurately, and avoid revisits by using some form of working memory to discriminate previously visited arms from unvisited arms (Olton \& Collison, 1979; Olton \& Samuelson, 1976; Suzuki, Augerinos, \& Black, 1980). Very high choice accuracy has been obtained in mazes with up to 17 arms (Olton, Collison, \& Werz, 1977), or even with 24 arms arranged in a hierarchical fashion (Roberts, 1979).

Perhaps the most salient aspect of rats' performance in the radial-arm maze is the resistance to treatments designed to impair choice accuracy. Delays of up to several hours have been interpolated during the choice sequence that constitutes each trial with little or no effect (Beatty \& Shavalia, 1980). During such delays, subjects have been given treatments such as sensory stimulation (Maki, Brokofsky, \& Berg, 1979), trials in other radial mazes (Cook \& Brown, 1985; Maki et al., 1979; Roberts, 1981), treatment with various drugs such as anesthetics (Beatty \& Shavalia, 1980) and amphetamines (Beatty \& Rush, 1983), and electroconvulsive shock (Maki, 1985; Shavalia, Dodge, \& Beatty, 1981). Although some of these treatments significantly impair performance under some conditions, in general they disrupt performance lit-

\footnotetext{
Support during the conduct of this research was provided by National Institute of Mental Health Grant MH42646. The author thanks Rae Force, Joe George, Jerry Gosnell, Lisa King, Louis Macero, Monica Melady, Bill McLaughlin, Grace Spagnola, and Myken Wilson for their assistance in conducting these experiments. Correspondence concerning this article may be addressed to Michael F. Brown, Department of Psychology, Villanova University, Villanova, PA 19085.
}

tle, if at all. The fact that choice accuracy in the radial maze is surprisingly impervious to so many such treatments undoubtedly accounts, in part, for the intense interest that this paradigm has received.

In the present series of experiments, a previously unexplored factor that has profound effects on radial-maze performance is introduced: the length of the maze arms. Typical radial mazes consist of arms approximately $80 \mathrm{~cm}$ in length. The present experiments include mazes with arms $37 \mathrm{~cm}$ in length.

\section{EXPERIMENT 1 LOW ACCURACY IN A MAZE WITH SHORT ARMS}

The first experiment was originally carried out for reasons other than those with which this report is concerned. It was designed to compare performance in a 16-arm radial maze with performance in a 12-arm maze. The 12-arm maze consisted of the 16-arm maze with four arms made inaccessible. Separate groups of rats were tested in these two maze configurations, first using a free-choice procedure and then using a two-alternative forced-choice (2AFC) procedure. The apparatus was constructed with unusually short arms.

\section{Method}

Subjects. The subjects were 32 experimentally naive, male Sprague-Dawley rats, approximately 4 months of age at the beginning of the experiment. They were maintained at $80 \%-85 \%$ of their free-feeding weights by supplemental feeding following each experimental session. The subjects were housed in groups of 4 and were transported in their home cages between the colony and experimental rooms daily. Experimental sessions were conducted during the dark phase of a 12:12 light:dark (LD) cycle.

Apparatus. The apparatus was a 16-arm radial maze constructed of $1.5-\mathrm{cm}$-thick plywood and painted white. The central platform 
was $60 \mathrm{~cm}$ in diameter. The arms were $10 \mathrm{~cm}$ wide and $37 \mathrm{~cm}$ in length, and extended from the central platform at equal angles. A gold-colored tin-foil ashtray ("E-Z foil" ; $6.0 \mathrm{~cm}$ in diameter and $1.0 \mathrm{~cm}$ deep) was attached to the end of each arm and served as the food cup. Walls were attached to one side of each arm to prevent the subjects from jumping from arm to arm. The walls were constructed of plywood (painted white), extended $19 \mathrm{~cm}$ from the central platform at a height of $12.5 \mathrm{~cm}$, and extended the remainder of the length of each arm at a height of $6 \mathrm{~cm}$. The maze was elevated $60 \mathrm{~cm}$ above the floor of the room. The apparatus is illustrated in the top panel of Figure 1.

The experiment was conducted in a $4.4 \times 3.1 \mathrm{~m}$ room. There was a bank of filing cabinets along one wall of the room, and additional cabinets and apparatus in the room. The room was illuminated by two fluorescent tubes.

Free-choice procedure. The rats were randomly assigned to one of two groups. Rats in the 16-arm group were run with free access to all 16 arms of the maze. Rats in the 12-arm group were run with access to four arms obstructed by wooden blocks constructed of 12.5 -cm lengths of " $2 \times 4$ " $(3.5 \times 8.5 \mathrm{~cm})$ painted black, which were placed at the entrance of each blocked arm. The blocked arms were separated from each other by three unblocked arms, and the same set of arms was blocked for each subject in the 12-arm condition.

During the initial five sessions, the subjects were shaped to obtain food from the food cups. They were placed, in groups of 4 ,
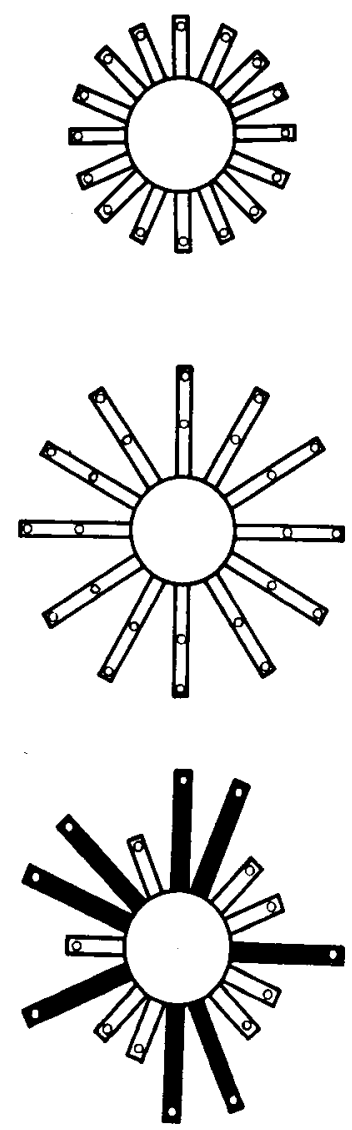

Figure 1. Scale drawings of the radial-arm mazes used in Experiment 1 (top panel), Experiment 2 (middle panel), and Experiment 3 (bottom panel). In the maze used in Experiment 2, foods cups were located in one of the two locations indicated, depending on the experimental group. on the maze with 45-mg sucrose pellets (BioServe, Inc.) scattered on the central platform and on the 16 (or 12) arms. Over the course of these five sessions, the number of pellets in the center of the maze and on the arms was reduced. By the fifth session, pellets were located only in the food cup.

Following these initial shaping sessions, the rats were run individually in the maze for one session per day. Prior to each session, two pellets were placed in each food cup. The rat was then placed in the central arena. A choice was defined by the rat passing the boundary between the taller and shorter sections of the wall along the arm (i.e., $19 \mathrm{~cm}$ from the center arena). The rat was allowed to choose arms until all 12 (or 16) arms had been visited, until twice as many choices had been made as there were arms (i.e., 24 for the 12-arm group or 32 for the 16-arm group), or until $12 \mathrm{~min}$ had elapsed. The subjects were run using this free-choice procedure for 60 sessions.

2AFC procedure. Following the completion of free-choice testing, the rats were tested using a 2 AFC procedure. Prior to each trial, some of the maze arms were blocked using blocks that were the same as those used to permanently block arms for the 12-arm group, except that they were painted white. In the 12-arm group, a randomly chosen set of $2,4,6,8$, or 10 arms was blocked on each trial (in addition to the 4 arms that were permanently blocked with the black blocks). In the 16-arm group, a randomly chosen set of $2,5,8,11$, or 14 arms was blocked on each trial. This manipulation of the point of delay interpolation (POI) was designed to conform to the procedure of Cook, Brown, and Riley (1985). The rat was placed in the maze and allowed to make choices until every unblocked arm had been visited. It was then removed from the maze and placed in a holding cage for $15 \mathrm{~min}$. When this retention interval had passed, all maze arms were blocked, except for one arm that had been visited before the delay and one unvisited arm. These arms were chosen randomly within the two categories with the constraint that they be either adjacent to each other or separated by only one arm (including the permanently blocked arms in the case of the 12-arm group). The rat was then placed in the maze and allowed to visit one of these two arms, with a visit to the formerly unvisited arm being considered correct and reinforced by two pellets in the food cup. The rat was then removed from the maze for approximately $5 \mathrm{sec}$ while the first pair of test arms was blocked and a second pair of test arms was unblocked. The members of this second pair consisted of one visited and one unvisited arm, randomly chosen with the same constraint used for the first test, and, in addition, the constraint that they could not be the same arms used in the first test. The rat was then allowed to visit one of the arms in this second test pair. If a choice did not occur within 3 min during either of the tests, the rat was removed from the maze and the session was terminated. Data from terminated sessions were not included in the analyses reported below.

Sessions were conducted in blocks of five, with each POI used once per block. One session per day was run. A total of eight blocks of sessions was conducted.

\section{Results}

Free-choice procedure. One rat in the 12-arm group consistently jumped from arm to arm by climbing over the walls separating the arms. Therefore, this rat was dropped from the experiment and its data are not included.

To determine changes in performance over the course of free-choice testing, the data were divided into blocks of 20 sessions each. The top and middle panels of Figure 2 show the mean percentage of sessions that resulted in the three possible outcomes: (1) successful completion [all 12 (or 16) arms visited], (2) removal following twice as many choices as there were arms of the maze ( 24 for the 12 - 


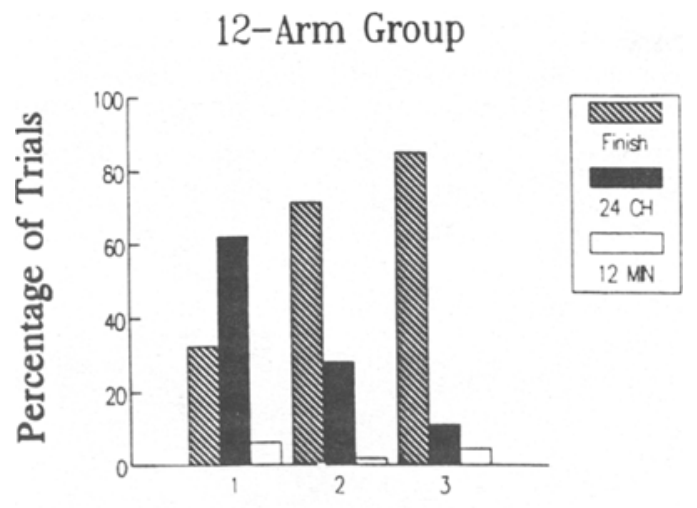

Blocks of 20 Sessions

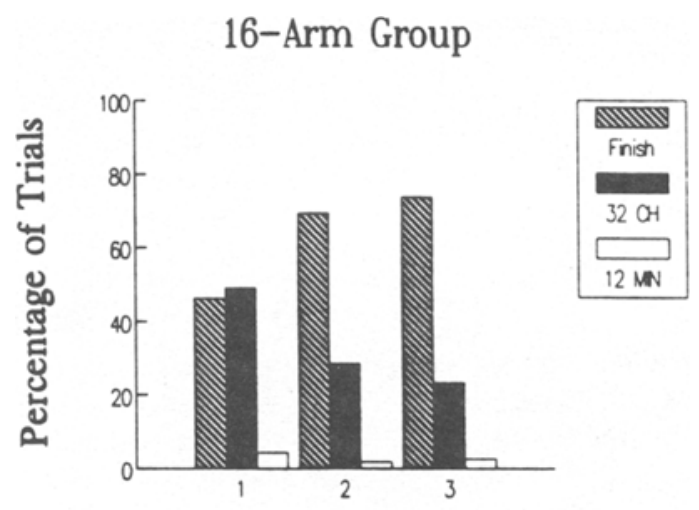

Blocks of 20 Sessions

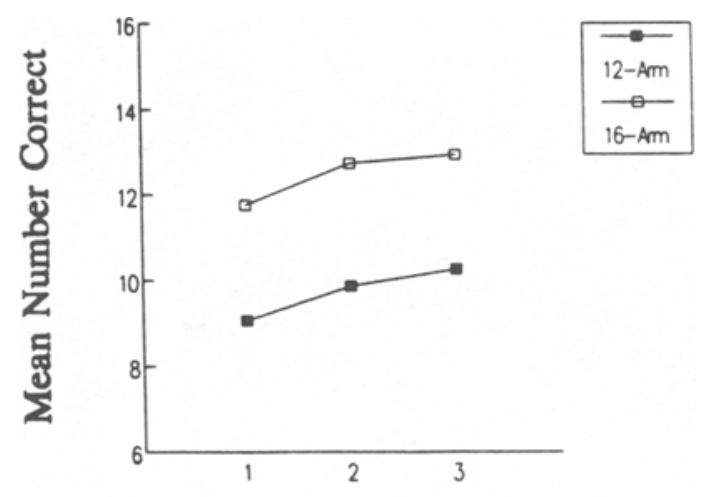

Blocks of 20 Sessions

Figure 2. Mean probability of the three possible trial outcomes over the course of the three blocks of free-choice sessions in Experiment 1 for the 12-arm group (top panel) and the 16-arm group (middle panel). The bottom panel shows the mean number of correct choices out of the first 12 (or 16) choices over the course of blocks for both groups.

arm group or 32 for the 16-arm group), and (3) removal following $12 \mathrm{~min}$ in the maze in the 12- and 16-arm groups, respectively. During the first block of sessions, about half of the sessions were terminated after 24 (or 32) total choices were made. An analysis of variance (ANOVA) was performed on the data from each group to compare the proportion of sessions successfully completed as a function of block. These analyses revealed that there was a significant improvement over the course of blocks for both the 12-arm group $[F(2,28)=67.3$, $p<.001]$ and the 16-arm group $[F(2,30)=19.0$, $p<.001]$.

The bottom panel of Figure 2 shows the mean number of correct choices out of the first 12 (12-arm group) or 16 (16-arm group). These means do not include sessions during which the rat was removed after $12 \mathrm{~min}$ had elapsed. ANOVAs revealed that there was a significant improvement over the course of blocks for both the 12arm group $[F(2,28)=33.5, p<.001]$ and the 16-arm group $[F(2,30)=13.6, p<.001]$.

An analysis was done to quantify the turning magnitudes associated with choices. This was done to determine the extent to which transitions from arm to arm were consistent and stereotypic (Brown \& Cook, 1986; Olton \& Samuelson, 1976; Suzuki et al., 1980). Each arm choice (except for the first choice of each session) was classified according to the number of arms separating it from the previously chosen arm. For example, choosing an arm immediately adjacent to the previously chosen arm resulted in a transition score of 1 . In the case of the 12arm group, blocked arms were counted in determining the transition score. That is, choosing an arm adjacent to one side of a blocked arm following a choice to the arm adjacent to the other side of the blocked arm resulted in a transition score of 2 .

The top panel of Figure 3 is a probability distribution of the turning magnitudes of the rats in the two groups during the last block of trials (i.e., Sessions 41-60). It shows that approximately $50 \%$ of all choices made were to an arm separated from the previously chosen arm by one, whereas approximately $25 \%$ were to an adjacent arm. If turning magnitudes were determined randomly, it would be expected that $13.3 \%$ of all choices would have turning magnitudes of $1,2,3,4,5,6$, and 7 , and $6.7 \%$ of all choices would have a turning magnitude of $8 .^{1}$

The bottom panel of Figure 3 shows the results of an analysis designed to measure the extent of individual biases for particular turn magnitudes. For each rat during each block of sessions, the modal turn magnitude was determined and the percentage of turns of that magnitude was calculated. Shown in the figure is the mean percentage of turns falling into the modal category as a function of group and block. ANOVAs revealed that there was a significant increase in this measure of turning-magnitude bias in the 12-arm group $[F(2,28)=5.0, p<.05]$ and a marginally significant decrease in turning bias in the 16-arm group $[F(2,30)=2.8, .1>p>.05]$.

Forced-choice procedure. Two rats (one from each group) were eliminated from the experiment during 2AFC testing because they consistently failed to make test choices within $3 \mathrm{~min}$.

Preliminary data analyses failed to produce any evidence that choice accuracy changed over the course of blocks. Therefore, a mean percentage of correct choices was calculated for each rat at each level of POI, collaps- 


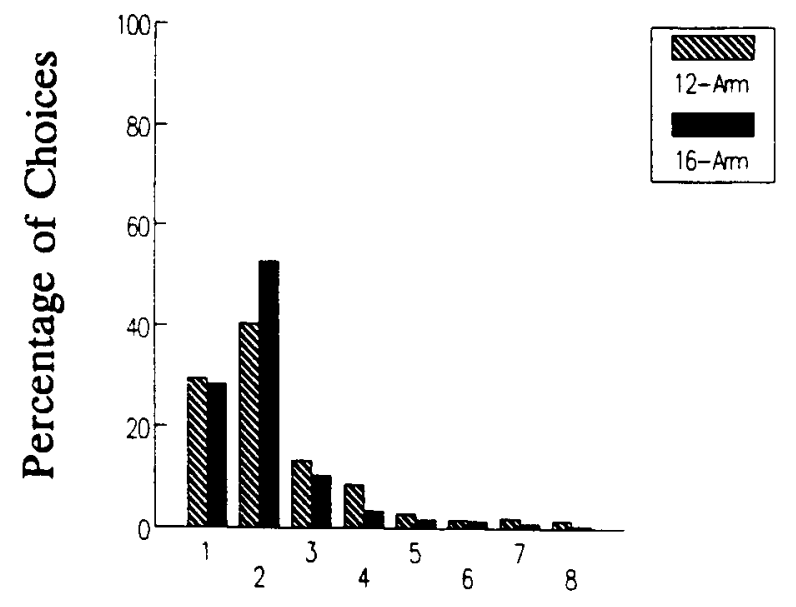

Turn Magnitude

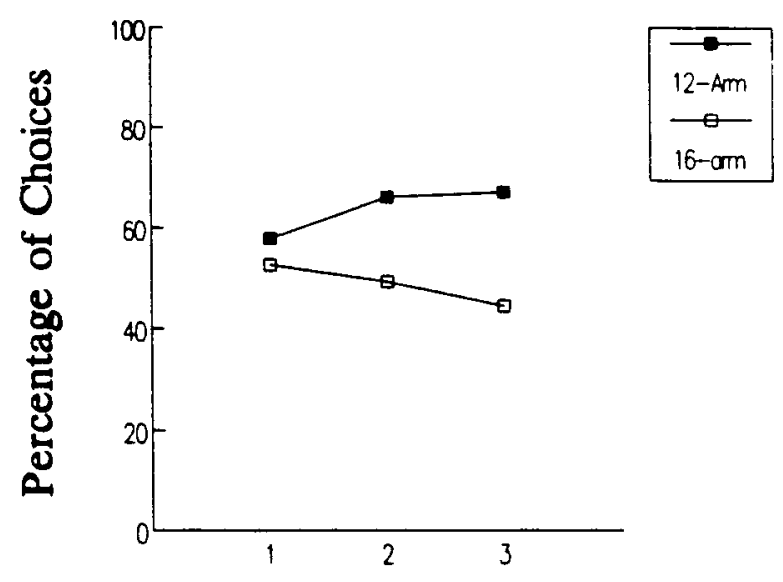

Blocks of 20 Sessions

Figure 3. Top panel: Distribution of turning magnitudes during the last block of free-choice sessions in Experiment 1. Bottom panel: Mean percentage of turns of the subject-specific modal turn magnitude over the course of the three blocks of sessions.

ing over blocks. Figure 4 shows the mean percentage of correct choices for the various conditions of the experiment. ANOVAs were conducted separately on the data of the 12- and 16-arm groups to determine if there was an effect of POI. In the case of the 12-arm group, there was a marginally significant effect of POI $[F(4,56)=$ $2.35, .05<p<.10$ ]. No evidence of an effect of POI was obtained in the case of the 16-arm group $[F(4,52)$ $=1.68]$. A second analysis was conducted to determine if choice-accuracy levels were significantly different from that expected on the basis of chance (50\%). The overall mean level of choice accuracy was determined for each rat and these values were compared to chance using $t$ tests. Choice accuracy was significantly different from chance for both the 12-arm group $[t(13)=6.44, p<.001]$ and the 16-arm group $[t(14)=2.73, p<.01]$.

\section{Discussion}

Although there was clear evidence of an improvement in choice accuracy over the course of free-choice testing, the levels of accuracy obtained contrasted sharply with those obtained in previous radial-maze experiments. Data from the 12-arm group of the present experiment are somewhat difficult to compare with those of other experiments because of the unusual technique of blocking four arms of a 16-arm maze to form a 12-arm maze. However, the results from the 16-arm group can be compared with those of several previous investigations. For example, Olton et al. (1977) found that rats made an average of 14.5 correct choices out of the first 17 choices in a 17-arm maze during Training Sessions 21-30. In an unpublished experiment from this laboratory, 15 rats in a 16 -arm maze with standard-length arms made a mean of 15.3 correct choices in the first 16 choices during Sessions $31-40$ of free-choice training (Brown, 1987). In sharp contrast, rats in the 16-arm group of the present experiment made a mean of 12.7 correct choices in the first 16 choices during Sessions 21-40. Furthermore, the failure of rats to complete the maze after twice as many choices as there were arms in the maze on a substantial number of trials is unprecedented.

In agreement with a number of previous findings (e.g., Brown \& Cook, 1986; Dale \& Innis, 1986; Foreman, 1985; Olton et al., 1977), the rats in the present experiment exhibited some degree of stereotypy in their choice behavior. The majority of choices made in both groups were to arms either adjacent to, or separated by a single arm from, the previous choice. Previous experiments have shown that even rats exhibiting marked consistency in turning magnitude sometimes demonstrate high levels of choice accuracy when procedures are introduced to prevent such biases (Dale \& Innis, 1986; Olton et al., 1977).

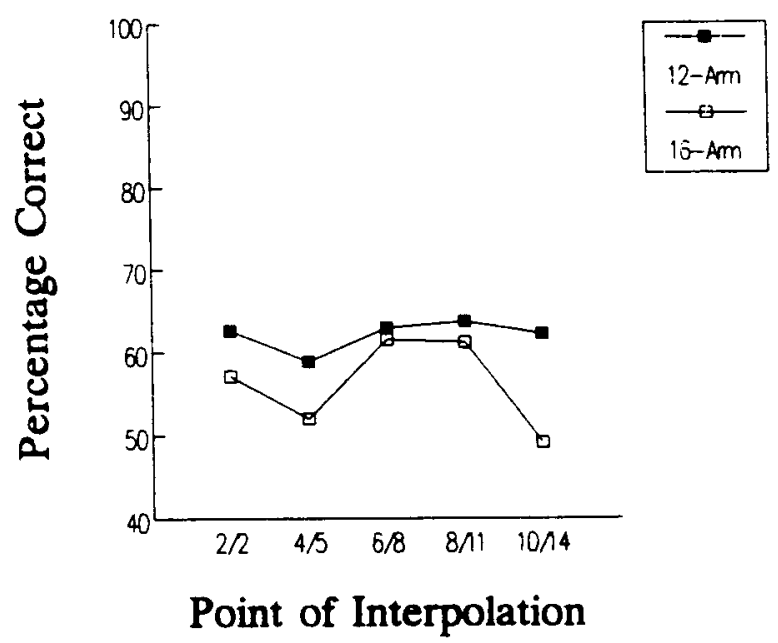

Figure 4. Mean percentage of correct choices in the two-alternative forced-choice tests of Experiment 1 for rats in the 12-arm and 16arm groups. 
The results of the $2 \mathrm{AFC}$ tests allow assessment of the ability to discriminate visited and unvisited arms when response stereotypy is prevented. Choice accuracy in the 2AFC procedure was significantly above that predicted on the basis of chance. However, it was substantially lower than that found in earlier experiments using similar test procedures. For example, Roberts and Smythe (1979) obtained 2AFC choice-accuracy levels of about $90 \%$ in an eight-arm radial maze. More directly comparable to the present data are those of Cook et al. (1985, Experiment 4 ), who used a procedure very similar to the present one and rats with a similar experimental history. They obtained choice-accuracy levels of approximately $90 \%$. Thus, although the rats in the present experiment performed at above-chance levels, choice accuracy was quite low relative to a large body of previous data.

\section{EXPERIMENT 2 \\ MANIPULATION OF MAZE-ARM LENGTH} IN A 12-ARM MAZE

Although the most salient difference between previously reported experiments using the radial maze and the experiment reported above is maze-arm length, clearly a direct comparison between performance in a maze with short and long arms is necessary to determine whether maze-arm length does, in fact, affect performance in the radial maze. In the present experiment, the choice behavior of two groups of naive rats, one trained in a maze with food cups at the ends of 80-cm-long arms and one trained in the same maze, but with the food cups $37 \mathrm{~cm}$ from the central arena of the maze, was compared. This allowed the performance of rats in a standard radial-arm maze to be directly compared with the performance of rats that ran only halfway down the $80-\mathrm{cm}$ arms to obtain food.

\section{Method}

Subjects. The subjects were 20 male Sprague-Dawley rats obtained from the same source and maintained in the same manner as the rats used in Experiment 1. They had previously participated in an experiment involving taste preferences, but were otherwise naive. They were approximately 6 months old when the experiment began.

Apparatus. The apparatus was a radial maze identical to that used in Experiment 1, with the following exceptions. First, the maze had 12 arms. Second, the arms were $80 \mathrm{~cm}$ in length. Third, a metal wall surrounded the central platform. A hole, $7.5 \mathrm{~cm}$ in diameter and centered $5.5 \mathrm{~cm}$ above the maze surface, allowed access to each arm. Metal doors in sliding tracks could be used to control access to each arm using a string-and-pulley system. Finally, the wooden walls mounted on one side of each arm were $25 \mathrm{~cm}$ long and $13 \mathrm{~cm}$ high. This apparatus is illustrated in the middle panel of Figure 1.

The experiment was conducted in a room immediately adjacent to the room used in Experiment 1. It was similar in most respects to the room used in the earlier experiment, and measured $4.3 \times$ $3.8 \mathrm{~m}$. The room was illuminated by a bank of four fluorescent tubes.

Procedure. The rats were randomly assigned to two groups with the constraint that there be 10 subjects in each group. Throughout the experiment, the food cups were located at the distal ends of the arms for rats in the long-arm group and $37 \mathrm{~cm}$ from the central arena for rats in the short-arm group. The rats were first shaped to enter the arms of the maze using the same procedure described above for Experiment 1.

Following shaping, 30 free-choice sessions were carried out. The doors to all 12 arms were open during these sessions. Two $45-\mathrm{mg}$ sucrose pellets were placed in each food cup. Each session consisted of a rat being placed in the central arena and allowed to make choices until all 12 arms had been visited, 24 choices had been made, or $10 \mathrm{~min}$ had elapsed. A choice was defined by the rat's nose crossing the plane defined by the end of the barrier mounted on the side of each arm. It should be noted that the rats in the short-arm condition never ventured beyond the food cups.

The rats were then tested using a forced-choice procedure designed to disrupt any response biases. At the beginning of each trial, the rat was placed in a fully baited maze with all 12 arms closed. After approximately $5 \mathrm{sec}$, the door to a single arm was opened. While the rat visited the first arm, the door to a second arm was opened. During the rat's visit to the second arm, the door to the first arm was closed and the door to a third arm was opened. This process was continued until the rat had visited a set of six randomly chosen arms. After its sixth arm visit, the rat was removed and placed in a holding cage for $15 \mathrm{~min}$. During each rat's delay, other rats were run in the maze. Following the delay, the rat was placed in the maze with all 12 doors open, and only those not visited prior to the delay were baited. The rat was allowed to visit arms until all six baited arms had been visited or 10 min elapsed. Trials were conducted using this procedure until each rat had successfully completed 10 trials (visited all six baited arms following the delay).

\section{Results and Discussion}

Free-choice trials. Data from the last 10 free-choice sessions are presented. Both groups attained high levels of choice accuracy. The rats in the short-arm group made a mean of 11.4 correct choices out of first 12 arm visits, whereas the rats in the long-arm group made a mean of 11.1 correct choices out of the first 12 . These values do not differ $[t(18)=.95]$. Clearly, the expectation based on the results of Experiment 1-that subjects in the shortarm condition would perform poorly-was not confirmed.

An examination of the distributions of turning magnitudes in the two groups makes it clear, however, that the high level of choice accuracy in the short-arm group does not necessarily indicate an ability to discriminate previously visited and unvisited arms. These turn-magnitude distributions are shown in the top panel of Figure 5. The rats in the short-arm group chose an adjacent arm with a significantly higher probability than did the rats in the long-arm group $[t(18)=5.57, p<.001]$.

The high probability of an adjacent-arm response in the short-arm group renders the high level of choice accuracy obtained difficult to interpret. The adjacent-arm response produces errorless performance independent of any ability to discriminate visited and unvisited arms: The rat simply visits adjacent arms until it has visited each arm in turn. Therefore, the high levels of choice accuracy attained by the rats in the short-arm group may not be dependent on working memory. The rats in the long-arm group, on the other hand, were not biased to choose adjacent arms. Therefore, the high levels of accuracy attained by this group cannot be explained in terms of turn magnitudes. Thus, only the rats in the long-arm group 


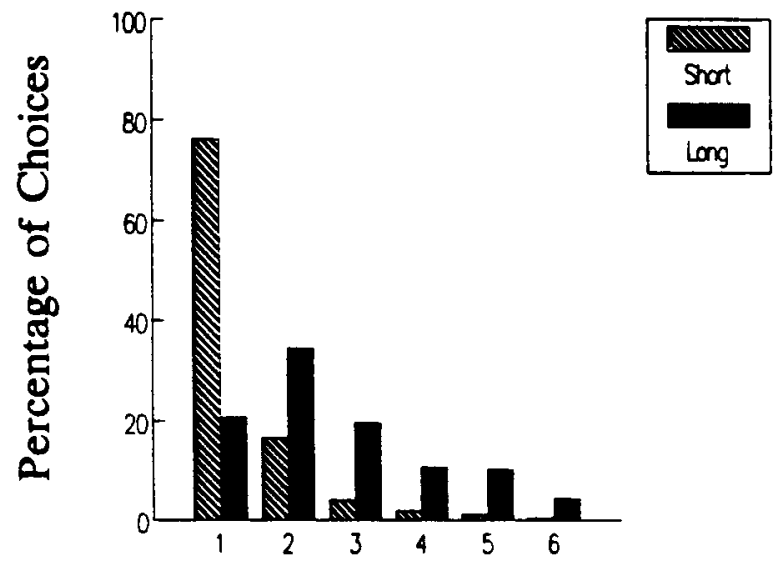

Turn Magnitude

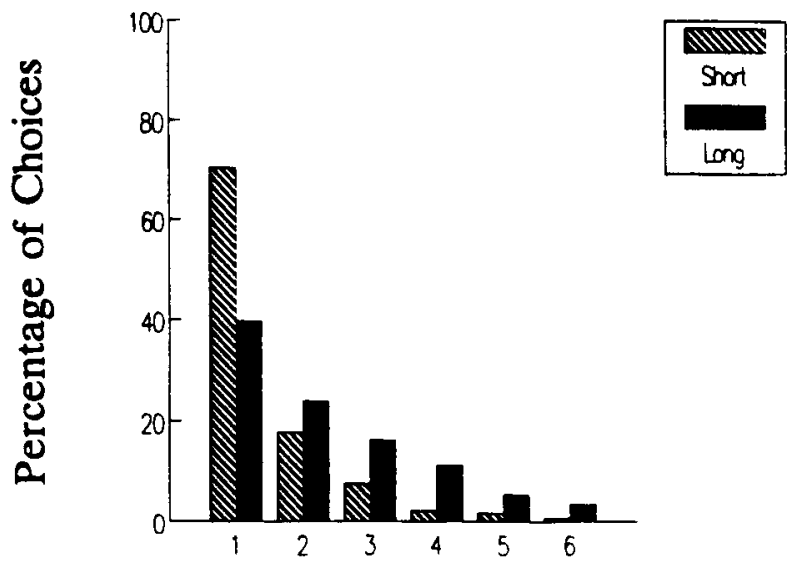

Turn Magnitude

Figure 5. Distributions of turning magnitudes during the last 10 free-choice sessions (top panel) and during the forced-choice sescions (bottom panel) of Experiment 2. Data from the forced-choice sessions include only choices made following the delay.

of the present experiment appeared to be accurately choosing arms on the basis of working memory.

In support of the hypothesis that the high level of choice accuracy attained by the short-arm group was the result of response stereotypy, there was a strong correlation between choice accuracy and the proportion of adjacent-arm responses among the rats in the short-arm group (Spearman's $e=.933, p<.001)$. This correlation was not present among the rats in the long-arm group $(\varrho=.433)$.

Forced-choice trials. In the forced-choice procedure, the first six choices are, by design, always correct. Therefore, the number of choices required to complete the maze, rather than the number of correct choices out of the first 12, will be reported. Rats in the short- and longarm groups required a mean of 15.6 and 13.45 choices, respectively, to complete the maze. These values were significantly different $[t(18)=3.90, p<.001]$. Thus, when the first six arms were chosen randomly, the rats in the long-arm condition were able to avoid subsequent revisits to those arms much more successfully than were the rats in the short-arm condition.

The bottom panel of Figure 5 shows the mean distribution of turn magnitudes for choices that occurred following the delay. As in the free-choice phase, the rats in the short-arm condition were much more biased to choose an arm adjacent to the previous choice than were the rats in the long-arm condition $[t(18)=4.92$, $p<.001]$. Thus, the bias to visit adjacent arms demonstrated by the rats in the short-arm condition persisted even when it resulted in a substantial number of errors. This is quite different from the results of previously reported experiments in which rats continued to exhibit high levels of choice accuracy when stereotypic response patterns were disrupted (Dale \& Innis, 1986; Olton et al., 1977).

\section{EXPERIMENT 3 \\ PERFORMANCE IN A 16-ARM MAZE WITH SHORT AND LONG ARMS}

The previous experiments show that performance in the radial-arm maze is strongly affected by the length of the maze arms. Relative to standard-length arms, short arms result in lower levels of accuracy and/or higher levels of response stereotypy.

The final experiment is an initial attempt to understand the mechanism producing these phenomena. Two classes of explanations for the effects of maze-arm length can be distinguished. The first attributes poor choice accuracy and/or response stereotypy to relatively poor working memory for short arms. That is, rats do not encode, store, and/or retrieve information that would allow the discrimination of previously visited and unvisited short arms. Discrimination ability might be diminished by the relatively smaller distance between the ends of the arms in mazes with shorter arms (thereby rendering the cues associated with the arms more similar in the short-arm maze), or perhaps by the fact that the wall separating the arms extended to the end of the arms (Experiment 1) or relatively close to the end of arms (Experiment 2) in the short-arm mazes used in these experiments. This might have reduced access to extramaze cues, which has been shown to reduce choice accuracy and increase response stereotypy (Innis \& Macgillivray, 1987; Mazmanian \& Roberts, 1983).

A second class of explanations points to nonmemorial processes that result in a relatively lax choice criterion for short arms. Rats may visit short arms for reasons that are independent of the quality of memory. Brown, Wheeler, and Riley (1989) recently introduced choicecriterion effects as an explanation for choice behavior in the radial-arm maze. They provided evidence that the choice criterion used by rats is more strict during choices made late in each trial than it is for choices made early. In addition, they argued that the criterion that determines whether an arm is visited (as opposed to continuing to investigate arms in the central arena) can and does in- 
fluence choice accuracy. In the present situation, the lowered levels of choice accuracy, as well as the higher levels of response stereotypy, could be explained as the result of a relatively lax criterion for visits to short arms as opposed to long arms.

The present experiment was designed to contrast these two classes of theories by using a 16-arm maze with eight short arms and eight long arms. With this apparatus, choices of long and short arms could be directly compared. A single group of rats was trained to perform in the maze, and their choice behavior was examined under both a free-choice procedure and a 2AFC testing procedure.

One critical issue addressed by this experiment was the extent to which initial (correct) visits to short arms would occur prior to initial visits to long arms in the free-choice procedure. A bias to visit short arms prior to visits to long arms in the free-choice procedure is predicted if there is a lower criterion for visiting short arms than long arms. A theory that attributes the results of Experiments 1 and 2 to differences in memory quality for short and long arms, on the other hand, would predict a higher probability of revisits to short arms than to long arms, but would not predict a tendency for initial visits to be directed toward short arms.

As a further test of the role of memory, rats were given 2AFC tests following the free-choice sessions. These tests provided the subject with either a choice between two short arms or a choice between two long arms. If a difference in the efficacy of working memory for long and short arms exists, then superior performance would be expected in the case of long-arm tests. The prediction of a criterion based theory is somewhat ambiguous. Although 2AFC tests have traditionally been used in part to rule out effects of choice criteria and bias, Brown et al. (1989) have pointed out that in the radial maze, rats are free to evaluate the two alternatives ad lib, as determined by the choice criterion. Because of this, choice accuracy in a 2AFC test could be affected by choice criterion.

\section{Method}

Subjects. Nine male rats from the same source and maintained in the same manner as the rats in the previous experiments served as subjects. They had earlier served in an experiment involving tastepreference learning, but were otherwise experimentally naive. They were approximately 6 months old at the onset of the present experiment.

Apparatus. The apparatus was the same 16-arm radial maze used in Experiment 1, modified in that eight randomly selected arms were lengthened to $80 \mathrm{~cm}$ by attaching plywood extensions to the short arms. Food cups were mounted at the ends of the arms. The long arms differed from the short arms not only in length, but also in that they were painted flat black. This apparatus is illustrated in the bottom panel of Figure 1.

Procedure. During the first five sessions of exposure to the maze, the shaping procedure described above for Experiment 1 was used.

Following shaping, the rats were given 20 sessions of free-choice testing. Prior to each session, two pellets were placed in each food cup. The rat was placed in the center of the maze and allowed to choose arms until each arm had been visited once, 31 choices had been made, or $10 \mathrm{~min}$ had elapsed.
The rats were then given 15 sessions of 2 AFC testing. Prior to each session, access to a randomly determined set of eight arms was obstructed using the same blocks used in Experiment 1. Two pellets were placed in the food cup of each unblocked arm. The rat was then placed in the center of the maze and allowed to visit arms until all eight unblocked arms had been visited. The rat was then removed from the maze and placed in an individual holding cage for $15 \mathrm{~min}$. Upon its return to the maze, all arms except two were blocked. One unblocked arm had not been visited prior to the delay and was baited with two pellets. The other unblocked arm had been visited prior to the delay and was unbaited. The rat was allowed to choose one of the two arms, or was removed from the maze if 10 min elapsed without a choice. The rat was then removed from the maze for approximately $5 \mathrm{sec}$ while the first set of two arms was blocked and a second set of two arms (one previously unvisited and one previously visited; both different than the arms used in the first test) was unblocked. The rat was then allowed to choose one of the arms from this second set. Within each test pair, the arms were of the same length. Thus, each 2AFC test consisted of either a choice between two short arms or a choice between two long arms.

\section{Results and Discussion}

Free-choice tests. The top panel of Figure 6 shows the mean probabilities of visiting short and long arms as a function of choice number during the 20 free-choice sessions. These probabilities sum to 1 prior to the 17 th choice, but are shown separately to depict the probability and nature of late choices. An ANOVA was used to determine if the probability of choosing a short arm changed over the course of the first 16 choices. This analysis revealed a significant effect of choice number $[F(15,120)=5.75, p<.001]$, due to a relatively stronger tendency for early (and therefore virtually always correct) choices to be directed to short arms. This shows clearly that there is a bias to visit short arms, independent of any difference in the ability to discriminate previously visited and unvisited arms as a function of arm length.

A second question to be asked of these data is whether revisits to short arms were more likely to occur than revisits to long arms. This analysis is complicated by the fact that most early visits were to short arms. A difference in the probability of revisits to short and long arms might be produced by the fact that more opportunities for short-arm revisits occurred by virtue of short arms having been visited earlier in the choice sequence. To unconfound the effects of arm length and ordinal position of choice on the probability of revisits, the probability of revisiting arms was calculated as a function of the ordinal position of the initial visit to that arm among the set of initial arm visits. The mean values for the 20 freechoice sessions are shown separately for revisits to long and short arms in the bottom panel of Figure 6. It should be noted that the values shown for long arms in the first few ordinal positions are based on very small $n s$. Despite this, it is quite clear that revisits to short arms were much more likely than revisits to long arms independently of the ordinal position of the initial visit to that arm. This was confirmed by an ordinal position $x$ arm length ANOVA performed on the means for individual subjects. 


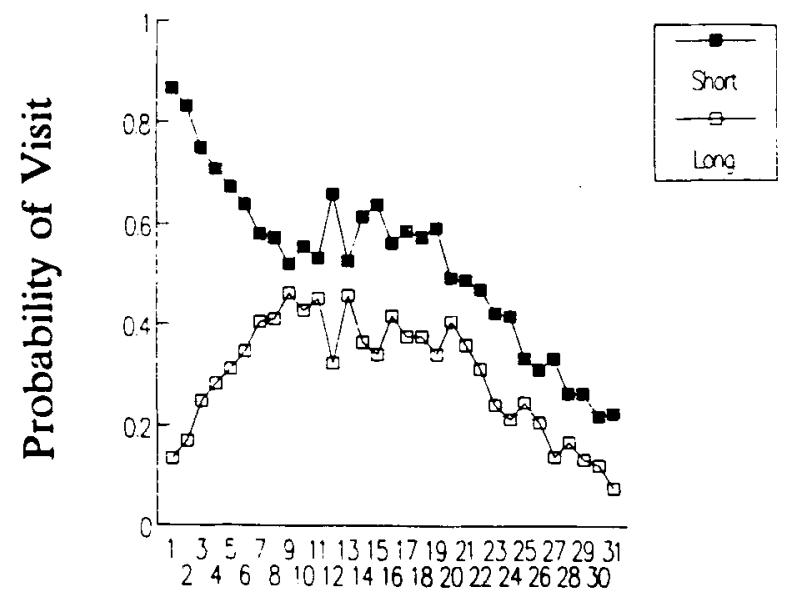

Choice Number

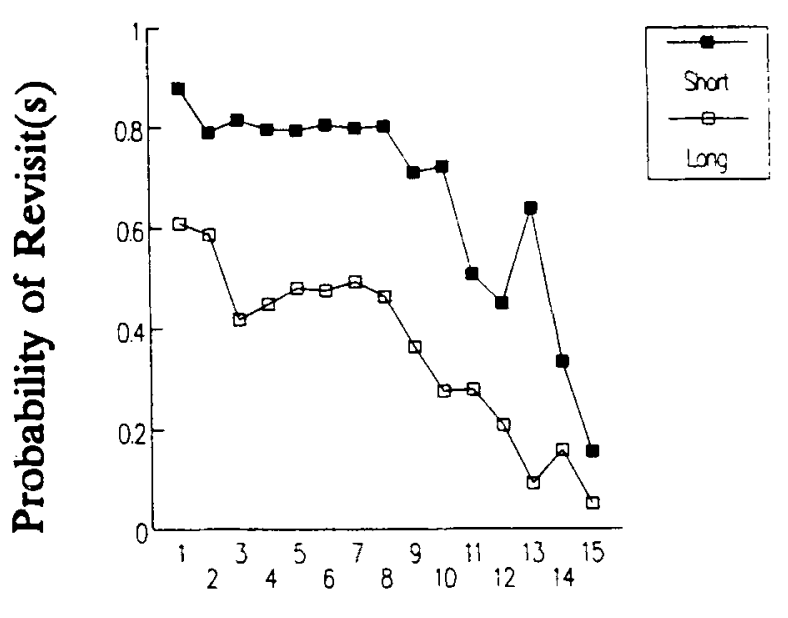

Ordinal Position Amons Initial Vivits

Figure 6. Top panel: Mean probability of visits to short and long arms as a function of choice number in Experiment 3. Bottom panel: Mean probability of one or more revisit(s) to each arm as a function of the ordinal position of the initial visit to that arm among the set of initial choices.

This analysis revealed an effect of ordinal position $[F(14,112)=16.5, p<.001]$ (which can be attributed simply to the decrease in opportunities to make errors as a function of the ordinal position of the initial choice) and an effect of arm length $[F(1,8)=83.9, p<.001]$. In addition, there was a marginally significant interaction between these variables $[F(14,112)=1.76, p=.054]$. The important result is that the rats were more likely to err by revisiting short arms than by revisiting long arms. This result could be explained either by poorer memory for short-arm visits than for long-arm visits or by a relatively lax choice criterion for short arms. That is, the effect of arm length on the probability of a revisit can be explained as resulting from the same mechanism that produced the effect of arm length on initial visits.

It is important to note that although there was a higher probability of revisits to short arms than to long arms, rates of revisits to long arms were also higher than would be expected on the basis of previous data. The reason for this apparent effect of the presence of short arms on revisits to long arms is not clear. It may be that there is some critical aspect of the structure of standard radialarm mazes that is disrupted by the use of different-length arms within the same maze. Alternatively, it may be that a lax choice criterion applied to short arms partially transferred to the long arms.

A tendency for particular arms or classes of arms to be visited early in the choice sequence has been previously reported (Batson, Best, Phillips, Patel, \& Gilleland, 1986; Melcer \& Timberlake, 1985). These preferences for certain arms have been produced by differences in the value of the reward associated with arms. The present finding extends the range of factors producing such effects to maze-arm length. Whether the subjects in the present experiment discriminated short arms from long arms on the basis of perception of the distance to the food cup, the brightness of the arm surface, the location of the arm, or some other cue cannot be determined from the present data.

2AFC tests. The mean percentage of correct choices in the 2AFC tests with short arms was $65.8 \%$, which was significantly greater than the chance level of $50 \%[t(8)$ $=3.30, p<.01]$. The mean percentage of correct choices in tests with long arms was $72.2 \%$, which was also significantly greater than chance $[t(8)=3.34$, $p<.01]$. However, these two values did not differ significantly from each other $[t(8)=1.02]$. Thus, the 2 AFC tests provide no evidence that there is a difference in the ability to discriminate previously visited and unvisited arms as a function of arm length.

As was the case in Experiment 1, the level of accuracy obtained in these $2 \mathrm{AFC}$ tests was substantially lower than that obtained in previously reported experiments (Cook et al., 1985). That this was true both in the case of shortarm tests and in the case of long-arm tests may reflect a global effect of the presence of short arms on choice accuracy. In any case, the lack of a difference between accuracy in short-arm tests and accuracy in long-arm tests should be interpreted with some caution, given that relatively low levels of accuracy were obtained in the longarm tests.

\section{GENERAL DISCUSSION}

These experiments introduce a relatively simple manipulation that has profound effects on spatial behavior. In the context of numerous reports of failure to disrupt choice behavior in the radial-arm maze with much more forceful treatments (e.g., Beatty \& Shavalia, 1980; Roberts, 1981), the robust effects produced by simply 
reducing the length of the maze arms by half are quite surprising. They underscore the need to determine more precisely the necessary and sufficient conditions for obtaining the high levels of choice accuracy that are typically found in this important experimental procedure.

Two distinct effects of arm length were apparent. In Experiments 1 and 3, and in the forced-choice phase of Experiment 2, short arms resulted in poor choice accuracy. In the free-choice phase of Experiment 2, short arms resulted in a very pronounced adjacent-arm response bias, which apparently accounted for the high level of choice accuracy that was obtained among the short-arm group. That the adjacent-arm response was responsible for high choice accuracy among the short-arm group is shown by the results of the forced-choice phase of Experiment 2 , in which response stereotypy was disrupted, resulting in low choice accuracy among the short-arm group relative to the long-arm group. In addition, there was a strong tendency for higher levels of choice accuracy to be associated with higher levels of adjacent-arm responding among rats trained in the short-arm maze.

The present experiments do not allow a determination of why high levels of adjacent-arm responding occurred in Experiment 2, but not in Experiment 1. One reasonable possibility is the difference in distance between the arms and/or the turn angles required in the mazes used in the experiments. In the 16-arm maze used in Experiments 1 and 3 , a sharper turn angle $\left(22.5^{\circ}\right)$ is required for an adjacent-arm response than is required in the 12 arm maze used in Experiment $2\left(30^{\circ}\right)$. It may be that this aspect of maze structure encouraged adjacent-arm responses in the 12-arm maze, but not in the 16-arm maze. The idea that turn angle may be important in determining the distribution of turn magnitudes has previously been suggested to explain the data of Yoerg and Kamil (1982), who found that a difference in the size of the central arena in an eight-arm maze produced differences in response stereotypy (Bond; Olton; cited in Yoerg \& Kamil, 1982).

Several lines of evidence from the present experiment show that although control of choice behavior by working memory was severely reduced by the use of short arms, it was not eliminated. First, choice accuracy improved substantially over the course of the free-choice sessions of Experiment 1. Although this improvement might be attributed to the changes in response stereotypy shown in the bottom panel of Figure 3, given that these changes were in opposite directions for the 12- and 16-arm groups, it seems more reasonable to attribute the improvement in accuracy to an increase in the use of discrimination ability. A second line of evidence that some level of discrimination ability remained when short maze arms were used comes from the 2AFC data of Experiments 1 and 3 . Although accuracy levels in those tests were relatively low, they were significantly above chance.

The mechanism producing the effects of maze-arm length is suggested by the results of Experiment 3, which was designed to contrast two classes of explanations: (1) that short arms lower the ability to discriminate previ- ously visited and unvisited arms, and (2) that a relatively lax choice criterion is applied to short arms. Two results from that experiment support the latter possibility. First, as predicted by the criterion-difference theory, there was a very strong bias for initial visits to be directed to short arms. Second, when given a choice between two short arms, rats chose the correct (previously unvisited) arm at a level of accuracy that did not differ significantly from accuracy in choosing between two long arms. This finding contradicts the theory that visits to long arms are better remembered than visits to short arms.

A lax choice criterion could explain the low levels of choice accuracy obtained in Experiments 1 and 3 and among the short-arm group in the forced-choice phase of Experiment 2. Brown et al. (1989) described in detail how differences in choice criterion can affect choice accuracy. In addition, it is quite reasonable to hypothesize that when choice behavior is not under the control of working memory, it is likely to be stereotyped, as it clearly was in Experiment 2. Thus, the most parsimonious interpretation of the present data, taken as a whole, is that short arms in the radial maze result in a lax choice criterion, which results in a reduction, but not elimination, of the control of choice behavior by working memory and a concomitant increase in the control of choice behavior by response stereotypy.

A relatively lax choice criterion may have resulted from any of several aspects of short maze arms. First, less time is required to reach the food cup following the choice of a short arm than following the choice of a long arm, thereby reducing the delay to reinforcement, a variable that is known to affect choice behavior (e.g., see Fantino, 1981). Second, less effort is required for a visit in the case of short arms. Thus, the energetic cost of arm visits is reduced. Cost/benefit analyses of choice behavior (e.g., Collier, 1982) would predict an increased tendency to visit arms under such conditions. Finally, casual observation of the early phases of training in the radial maze suggests that a certain amount of fear is associated with arm visits (presumably because of the elevation of the arms), and this would be reduced when arms are shortened. Which of these factors affect the choice criterion remains to be determined.

\section{REFERENCES}

Batson, J. D., Best, M. R., Phillips, D. L., Patel, H., \& Gilleland, K. R. (1986). Foraging on the radial-arm maze: Effects of altering the reward at a target location. Animal Learning \& Behavior, 14, 241-248.

Beatty, W. W., Rush, J. R. (1983). Retention deficit after damphetamine treatment: Memory deficit or performance change? Behavioral \& Neural Biology, 37, 265-275.

BeatTy, W. W., \& Shavalua, D. A. (1980). Spatial memory in rats: Time course of working memory and effect of anesthetics. Behavioral \& Neural Biology, 28, 454-462.

Brown, M. F. (1987). The effect of point of delay interpolation in a 16-arm radial maze. Unpublished manuscript.

Brown, M. F., Cook, R. G. (1986). Within-trial dynamics of radial maze performance in rats. Learning \& Motivation, 17, 190-205.

Brown, M. F., Wheeler, E. A., Riley, D. A. (1989). Evidence 
for a shift in the choice criterion of rats in a 12-arm radial maze. Animal Learning \& Behavior, 17, 12-20.

Collier, G. H. (1982). Determinants of choice. In D. J. Berstein (Ed.), Nebraska symposium on motivation, 1981: Response structure and organization (pp. 69-128). Lincoln: University of Nebraska Press.

CoOK, R. G., Brown, M. F. (1985). Retroactive interference in rat radial maze performance: The role of point of delay interpolation and the similarity and amount of interpolated material. Animal Learning \& Behavior, 13, 116-120.

Cook, R. G., Brown, M. F., \& Riley, D. A. (1985). Flexible memory processing by rats: Use of prospective and retrospective information in the radial maze. Journal of Experimental Psychology: Animal Behavior Processes, 11, 453-469.

DALE, R. H. I., \& INNIS, N. K. (1986). Interaction between response stereotypy and memory strategies on the eight-arm radial maze. $B e$ havioural Brain Research, 19, 17-25.

Fantino, E. (1981). Contiguity, response strength, and the delayreduction hypothesis. In P. Harzem \& M. D. Zeiler (Eds.), Advances in analysis of behavior: Vol. 2. Predictability, correlation, and contiguity (pp. 169-201). New York: Wiley.

Foreman, N. (1985). Algorithmic responding on the radial maze in rats does not always imply absence of spatial encoding. Quarterly Journal of Experimental Psychology, 37B, 333-358.

InNIS, N. K., \& MACGILlivray, M. (1987). Radial maze performance under food and water deprivation. Behavioural Processes, 15, 167-179.

MAKI, W. S. (1985). Differential effects of electroconvulsive shock on concurrent spatial memories: "Old" memories are impaired while "new" memories are spared. Behavioral \& Neural Biology, 43, $162-177$.

MAKI, W. S., BrokofSky, S., \& Berg, B. (1979). Spatial memory in rats: Resistance to retroactive interference. Animal Learning \& Behavior, 7, 25-30.

Mazmanian, D. S., \& Roberts, W. A. (1983). Spatial memory in rats under restricted viewing conditions. Learning \& Motivation, 14, 123-139.

Melcer, T., \& Timberlake, W. (1985). Poison avoidance and patch (location) selection in rats. Animal Leaming \& Behavior, 13, 60-68.
OLton, D. S., Colluson, C. (1979). Intramaze cues and "odor trails" fail to direct choice behavior on an elevated maze. Animal Learning \& Behavior, 7, 221-223.

Olton, D. S., Collison, C., \& Werz, M. A. (1977). Spatial memory and radial arm maze performance of rats. Learning \& Motivation, 8, 289-314.

Olton, D. S., \& SAmuelson, R. J. (1976). Remembrance of places past: Spatial memory in rats. Journal of Experimental Psychology: Animal Behavior Processes, 2, 97-116.

RoBERTS, W. A. (1979). Spatial memory in the rat on the hierarchical maze. Learning \& Motivation, 10, 117-140.

ROBERTS, W. A. (1981). Retroactive inhibition in rat spatial memory. Animal Learning \& Behavior, 9, 566-574.

Roberts, W. A., \& SMYThe, W. E. (1979). Memory for lists of spatial events in the rat. Learning \& Motivation, 10, 313-336.

Shavalia, D. A., Dodge, A. M., \& Beatty, W. W. (1981). Timedependent effects of ECS on spatial memory. Behavioral \& Neural Biology, 31, 261-273.

Suzuki, S., Augerinos, G., Black, A. H. (1980). Stimulus control of spatial behavior on the eight-arm maze in rats. Leaming \& Motivation, 11, 1-18.

YoerG, S. I. \& KamiL, A. C. (1982). Response strategies in the radial arm maze: Running around in circles. Animal Learning \& Behavior, $10,530-534$

\section{NOTE}

1. In this and all analyses of turning-magnitude distributions, expected frequencies are based on the fact that there are two arms separated by each turning magnitude except for the largest magnitude. The analyses ignore returns to the arm just visited (magnitude $=0$ ), which virtually never occurred.

(Manuscript received February 7, 1989; revision accepted for publication June 15, 1989.) 